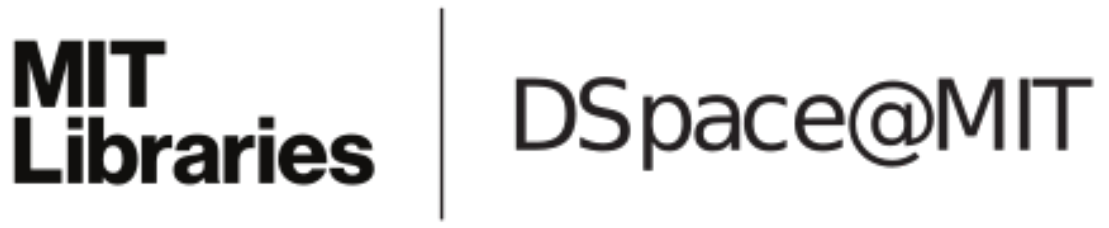

\author{
MIT Open Access Articles
}

Risk-Driven Design Processes: Balancing

Efficiency with Resilience in Product Design

The MIT Faculty has made this article openly available. Please share how this access benefits you. Your story matters.

Citation: Oehmen, J., and W. Seering. "Risk-Driven Design Processes: Balancing Efficiency with Resilience in Product Design." The Future of Design Methodology. Ed. Herbert Birkhofer. 44-54. Springer-Verlag, 2011.

As Published: http://dx.doi.org/10.1007/978-0-85729-615-3_4

Publisher: Springer-Verlag

Persistent URL: http://hdl.handle.net/1721.1/79871

Version: Author's final manuscript: final author's manuscript post peer review, without publisher's formatting or copy editing

Terms of use: Creative Commons Attribution-Noncommercial-Share Alike 3.0 


\title{
Risk-Driven Design Processes: Balancing Efficiency with Resilience in Product Design
}

\section{Josef Oehmen and Warren Seering}

Massachusetts Institute of Technology, Department of Mechanical Engineering, Room 3-471, 77 Massachusetts Avenue, Cambridge, MA 02139, USA

Email: oehmen@mit.edu, Phone: +1-617-452-2604

Email: seering@mit.edu, Phone: +1-617-253-3979

Published as: Oehmen, J. and Seering, W.: Risk-Driven Design Processes - Balancing Efficiency with Resilience in Product Design. In Birkhofer, H., ed. The Future of Design Methodology, p. 47-54 (Springer, London, 2011). DOI: 10.1007/978-0-85729-615-3_4

\begin{abstract}
Current design methods and approaches focus on increasing the efficiency of the product design system by, for example, eliminating waste and focusing on value creation. However, continuing failures in the development of complex, large scale products and systems point towards weaknesses in the existing approaches. We argue that product development organizations are hindered by the many uncertainties that are inherent in the process. Common management heuristics ignore uncertainty and thus overly simplify the decision making process. Creating transparency regarding uncertainties and the associated risks (i.e. effect of uncertainties on design objectives) is not seen as an explicit priority. Consequently organizations are unable to balance risk and return in their development choices. Product development processes do not emphasize reduction of risks, particularly those risks that are apparent early in the process. In addition, the resilience of the PD system, i.e. its ability to deliver on-target results under uncertainty, is not deliberately designed to match the level of residual uncertainty. This chapter introduces the notion of Risk-Driven Design and its four principles of 1 . Creating transparency regarding design risks; 2. Risk-driven decision making; 3 . Minimizing uncertainty; and 4. Creating resilience.
\end{abstract}

\section{The challenges of complex product design projects}

The development of complex products and systems continues to pose significant challenges for companies. For example, the US Aerospace \& Defense industry (GAO 2010) is facing massive cost and schedule overruns by $45 \%$ and 22 months respectively. This results in a projected cost overrun of $\$ 296$ billion for the largest 96 systems that are currently being developed (which is more than the annual GDP of South Africa, Finland, or Portugal). These problems persist, although there is a host of product design and development processes and methods that place significant emphasis on high efficiency (Krishnan and Ulrich 2001), i.e. generating a high-performing product or service with minimum cost and within minimum time.

Conventionally, emphasis is placed on increasing the efficiency of the design process, that is minimizing the amount or quality of input factors while maximizing the output. The relevant attributes of the output (i.e. product and/or service) are characterized by the trade-off among different objectives (Griffin and Page 1996), such as time (e.g. design project schedule, time to market), cost (e.g. 
design project budget, unit production cost) and quality (e.g. product performance or reliability). The overall goal is to make effective trade-offs among quality, cost, and time, both in terms of efficiency - reaching a higher overall 'average' among the objectives - as well as in terms of explicitly strengthening one of the objectives at the expense of the others. While some approaches acknowledge uncertainty and probability in the way that the achievement of performance objectives is modeled (most notably Design for Six Sigma), PD projects are generally planned based on static and 'foreseeable' point estimates.

\section{Uncertainty and Risk in Product Design}

Uncertainty and uncertainty reduction are at the heart of every product development and design project. Designers generate information, transform imprecise into precise information, and gradually reduce uncertainty (Sommer et al. 2008). The theory of bounded rationality explains how the decision making of individuals is always subject to uncertainty (Simon 1997). Extending this theory to organizations (for example in the theory of costly rationality, Radner 2000), it becomes apparent that in team and group settings uncertainty is even more relevant due to costly (and therefore limited) communication processes that are necessary to reduce it. There is a rich literature stream attempting to classify and describe different types of uncertainties (Halpern 2005; Knight 1964; Lindley 2006; Morgan and Henrion 1992; Paté-Cornell 1996; Taleb 2010; Pich et al. 2002; Chalupnik et al. 2009; de Weck and Eckert 2007; McManus and Hastings 2005). These classifications for example include the three categories of 'known uncertainties' (known probability distribution), 'Knightian uncertainty', or 'ambiguity' (unknown probability distribution) and 'Black Swans', 'wicked problems' or 'unknown unknowns' (unforeseeable uncertainty, i.e. even the fact that there is an uncertainty in the first place is not known). Other classifications for example differentiate regarding the origin of the uncertainty, e.g. factors endogenous to the PD process (technology and process execution) and exogenous factors (process environment: market, user, culture)

Following the ISO 31000, we understand risks as the "effect of uncertainty on objectives" (ISO 2009). Risks are therefore defined as the quantified impact of uncertainties on the objectives of the PD project. This general definition of risk can be concretized in a number of ways, their merit depending on the circumstances and goals of the risk analysis (see for example Kaplan and Garrick 1981; Haimes 2009; Paté-Cornell 1996). The underlying mental model is that of probability-distributed input factors leading to a probability-distributed achievement of objectives. In this context, risk can be understood in various ways:

- as the probability of failure to achieve a specific target;

- as the maximum possible deviation from an objective for a project;

- as a probability-weighted deviation from an objective, either as a point estimate of a single impact and probability pair or as the integration of a probability-distributed objective function; or

- as the variance from a mean value for achieving an objective.

As risks are functions of both the uncertainty of input factors and their impact on PD objectives, a number of risk taxonomies are possible. They can be structured along the input factors (e.g. technology risk, process risk, customer requirements risk), or along the objectives that they impact (e.g. cost risk, schedule risk, performance risk). Also, risks are linked in complex causal networks (e.g. technology uncertainty leading to schedule slip leading to cost overrun), making it often necessary to 
discuss them as part of larger cause-and-effect networks of risks, where uncertainties propagate over several levels until impacting an objective, which may in turn impact other objectives.

In the following, we give some examples of risks in product design structured along uncertainty in the input factors of the PD system. As uncertainty can affect every element of product design, this list is necessarily incomplete:

- Company-internal uncertainties: Uncertainty exists regarding the effectiveness of new development processes (e.g. ability of review processes to catch errors). Uncertainty also arises in communication processes regarding both the scope (completeness) as well as the quality (correctness) of communicated information. This is related to uncertainty in the coordination of work among individuals or groups. Uncertainty may also arise regarding the overall status and progress of the project. This leads to uncertainty regarding planning and forecasting, which determines the level of uncertainty of target levels for different objectives. Uncertainty surrounding the capabilities and productivity in engineering has a significant effect on cost, schedule and performance.

- Supplier uncertainties: As significant parts of the value creation in all life cycle phases are executed by suppliers, uncertainties regarding supplier performance during the development process can cause performance, schedule as well as cost risks.

- Customer requirements uncertainties: Both customers' uncertainty regarding their needs and the uncertainty with which these needs are understood by the project team have significant impact on the project performance.

- Market uncertainties: Environmental factors, such as demographic changes (e.g. aging population) or social trends (e.g. concerns regarding global warming), as well as actions by competitors (e.g. pricing strategy or new technology introduction) can significantly alter target specifications.

- Technology: Technology uncertainty affects an array of product outcomes. For example, technology maturity affects the performance reliability under field conditions. Systems integration maturity affects overall system performance and reliability. Production system maturity affects cost and lead time for manufacturing, and service system maturity affects operation and maintenance cost.

Risk management processes and methods have emerged as distinct management practices and foci of research (Oehmen et al. 2010; Sommer et al. 2008). Based on the above discussion as well as a review of the risk management literature, we advocate the integration of these methods directly into the design process via Risk-Driven Design, described below.

\section{Risk-based View: Risk-Driven Design}

Risk-Driven Design places a different emphasis on the management of the design process than conventional efficiency-driven design (see Figure 1). When the design process is driven by the intention to manage risk, uncertainties and their effect on the objectives are identified and quantified. Decision making then focuses on risks, usually the most critical first. This is done by reducing the level of uncertainty as much as reasonable and then creating a resilient PD system that can absorb the residual uncertainty to achieve the objectives within the target range. 


\begin{tabular}{|c|c|}
\hline $\begin{array}{l}\text { Principle } 1 \\
\text { Creating Transparency } \\
\text { Regarding Design Risks }\end{array}$ & $\begin{array}{l}\text { - Explore and identify knowable uncertainties } \\
\text { - Quantify resulting risks }\end{array}$ \\
\hline $\begin{array}{l}\text { Principle } 2 \\
\text { Making Risk-Driven } \\
\text { Decisions }\end{array}$ & $\begin{array}{l}\text { - Resource allocation to retire biggest risks first } \\
\text { - Objective setting associated with risk assessment } \\
\text { - Entrepreneurial decision making based on risk-return analyses }\end{array}$ \\
\hline $\begin{array}{c}\text { Principle } 3 \\
\text { Minimizing Uncertainty in } \\
\text { Design }\end{array}$ & $\begin{array}{l}\text { - Reduction of external uncertainty } \\
\text { - Reduction of internal uncertainty }\end{array}$ \\
\hline $\begin{array}{l}\text { Principle } 4 \\
\text { Creating Resilience in the } \\
\text { Design System }\end{array}$ & $\begin{array}{l}\text { - Create agile design system } \\
\text { - Create critical buffers in the design system }\end{array}$ \\
\hline
\end{tabular}

Fig. 1. The four principles of risk-driven design

\section{Principle 1: Creating Transparency Regarding Design Risks}

The first step in creating transparency regarding design risks is the identification of uncertainties. The second step is to bring clarity to understanding of the identified uncertainties by quantifying them. This can be done to different degrees. Purely verbal descriptions of uncertainties without the possibility for quantification represent the lowest level of knowledge about an uncertainty. Point estimates of uncertainties and their consequences represent the next higher level of fidelity, for example a $30 \%$ probability of a $\$ 1$ million penalty payment. Continuous probability distributions - for example regarding workforce productivity - offer the greatest amount of information. However, all descriptions of uncertainty are only as reliable as the input data they are based on, whether it is expert opinions, simulation models, or historic data with limited applicability to the future.

\section{Principle 2: Making Risk-Driven Decisions}

Having transparency regarding the different risks in a project yields several benefits in itself:

Transparency allows managers to prioritize their time and resource allocation to address the largest risks first.

When objectives are set, transparency enables the decision makers to consider the associated probability of success. This helps to set more realistic objectives. It also helps to ensure that planning forecasts (e.g. cost and schedule estimates) are all based on the same confidence level and therefore comparable.

Similarly, when trade-offs are made, decision makers have an additional degree of freedom: Every trade-off between objectives is associated with a corresponding level of risk - specifying high cost and long lead time for a complex system is much less risky than targeting the same system to be a low-cost, short lead time development. It becomes transparent how ambitious a certain set of objectives is.

Transparency enables determination of risk-return trade-offs, creating the opportunity for entrepreneurial decision making: High risk - high return options (e.g. choice of high performing new technol- 
ogy) can be balanced with low risk - low return options (e.g. established medium-performance technology), low risk - high return options can be favored and high risk - low return options eliminated. This will yield a balance of risk taking and performance between the different components of a product and/or service.

This will ultimately allow creation of better design project portfolios on the corporate level, as 'risky' high risk - high return projects can be balanced with 'sure bets' of low risk - low return projects to achieve an overall optimum risk-return balance for the company.

\section{Principle 3: Minimizing Uncertainty in Design}

In order to actively manage risks, managers have two fundamental options: reducing the uncertainty underlying risks and therefore their underlying causes, or making the PD system resilient against uncertainty, that is enabling it to achieve its objectives given a range of input factors.

As discussed above, uncertainty can be reduced, but never completely removed. In the case of product design, we argue that most engineering activities focus on the reduction of uncertainty, and Risk-Driven Design provides a framework for a more guided and focused uncertainty reduction.

The reduction of the overall risk exposure of a project can be used as an important key performance indicator, to incentivize retiring significant risks as early as possible in the design process, instead of succumbing to the (natural) inclination of postponing dealing with them as long as possible, leading to sudden deviations from objectives such as delays and cost overruns during the late project phases.

\section{Principle 4: Creating Resilience in the Design System}

Uncertainties can be reduced to some extent; some easily and cost efficiently (e.g. internal uncertainties due to factors that are under the direct control of the company); some with more effort (e.g. external uncertainties from the project environment). Some cannot be influenced at all (e.g. natural catastrophes or residual uncertainty due to bounded rationality). At any rate, every product design project will be facing a residual amount of uncertainty that must be dealt with. The ability of the PD system to deal with this uncertainty can be broken down into two categories: agility, i.e. the ability to respond effectively to unexpected events (Chalupnik et al. 2009), and robustness, i.e. mechanisms to absorb process deviations so that project outcomes remain within the target range, e.g. through buffers.

Agility describes the ability of the PD system to deliver stable performance under varying circumstances. This includes swiftness, the ability to detect errors quickly and plan and take corrective action, for example through unbureaucratic change management; cost efficiency, the ability to accommodate changes at low cost, for example through properly aligned organizational and supply chain incentives; flexibility, the ability to change objectives, for example through regular customer integration and consultation; and versatility, the ability of the PD system to process unexpected challenges, for example due to a broadly skilled workforce or adaptable development processes.

Buffers can fall into several categories: There can be buffering for each objective, e.g. financial buffers (more financial reserves than needed according to plan), schedule buffers (schedule reserves), 
and performance buffers (e.g. redundancy or overengineering). Buffers can also be created at lower levels, for example by holding excess capacity (e.g. testing facilities).

While creating an agile PD system aligns very well with efficiency-driven management styles, the creation of buffers does not. Transparency regarding the projects risk situation forms the basis for making a business case in favor of establishing critical buffers, and against excess buffers.

\section{Research Agenda}

The concept of risk-driven design needs both additional theoretical and empirical research if it is to mature. From a theoretical standpoint, a gap- and overlap-free taxonomy of design uncertainties and risks has to be developed. Also, risk-driven design has to be understood in the context of various design and product development frameworks, for example stage gate models, spiral development, $\mathrm{V}$ - or waterfall-models, concurrent engineering, or set-based design. Similarly, the risk management literature has to be explored to transfer relevant processes and methods into risk-driven design. There is also a rich literature stream regarding decision making under uncertainty, as well as the psychological dimension of human perception and treatment of risk and uncertainty that will have to be addressed.

Empirical research has to be done to uncover the current state of practice regarding the treatment of risk and uncertainty in industry. The viability of the four principles of risk-driven design has to be tested in both in-depth case studies and longitudinal surveys.

Acknowledgments The authors thank Alison Olechowski, Eric Rebentisch, Mohamed Ben-Daya and Günter Lessing for their thoughtful comments, as well as the Lean Advancement Initiative (LAI) at MIT and the MIT-KFUPM Center for Clean Water and Energy for their funding support.

\section{References}

Chalupnik MJ, Wynn DC, Clarkson PJ (2009) Approaches to mitigate the impact of uncertainty in development processes. Proceedings of the International Conference on Engineering Design, ICED'09, 24-27 August, Stanford, CA

de Weck O, Eckert C (2007) A classification of uncertainty for early product and system design. MIT Engineering System Division - Working Paper Series - ESD-WP-2007-10

GAO (2010) Defense Acquisitions - Managing Risk to Achieve Better Outcomes (GAO 10-347T). United States Government Accountability Office, Washington, D.C.

Griffin A, Page AL (1996) PDMA Success Measurement Project: Recommneded Measures for Product Development Success and Failure. Journal of Product Innovation Management 13 (6):478-496

Haimes YY (2009) Risk Modeling, Assessment, and Management. $3^{\text {rd }}$ edn. Wiley, Hoboken, NJ Halpern JY (2005) Reasoning about Uncertainty. MIT Press, Cambridge, MA

ISO (2009) ISO 31000:2009(E) - Risk management - Principles and guidelines. International Organization for Standardization, Geneva

Kaplan S, Garrick BJ (1981) On the quantitative definition of risk. Risk Analysis 1 (1):11-27

Knight FH (1964) Risk, Uncertainty and Profit. Augustus M. Kelley, New York, NY

Krishnan V, Ulrich KT (2001) Product Development Decisions: A Review of the Literature.

Management Science 47 (1):1-21

Lindley DV (2006) Understanding Uncertainty. Wiley, Hoboken, NJ 
McManus H, Hastings D (2005) A Framework for Understanding Uncertainty and its Mitigation and Exploitation in Complex Systems. Fifteenth Annual International Symposium of the International Council on Systems Engineering (INCOSE), 10 July to 15 July 2005, Rochester, NY

Morgan MG, Henrion M (1992) Uncertainty: A Guide to Dealing with Uncertainty in Quantitative Risk and Policy Analysis. Cambridge University Press, Cambridge, UK

Oehmen J, Ben-Daya M, Seering W, Al-Salamah M (2010) Risk Management in Product Design: Current State, Conceptual Model and Future Research. Proceedings of the ASME 2010 International Design Engineering Technical Conferences \& Computers and Information in Engineering Conference IDETC/CIE 2010

Paté-Cornell ME (1996) Uncertainties in risk analysis: Six levels of treatment. Reliability Engineering \& System Safety 54 (2-3):95-111

Pich MT, Loch CH, de Meyer A (2002) On Uncertainty, Ambiguity, and Complexity in Project Management. Management Science 48 (8):1008-1023

Radner R (2000) Costly and bounded rationality in individual and team decision-making. Industrial and Corporate Change 9 (4):623-658

Simon HA (1997) Administrative behavior: a study of decision-making processes in administrative organizations. $4^{\text {th }}$ edn. Free Press, New York

Sommer SC, Loch CH, Pich MT (2008) Project risk management in new product development. In: Loch CH, Kavadias S (eds) The Handbook of New Product Development Management. Elsevier, Oxford, pp 439-465

Taleb NN (2010) The Black Swan: The Impact of the Highly Improbable. $2^{\text {nd }}$ edn. Random House, 